\title{
A Role for Arabidopsis miR399f in Salt, Drought, and ABA Signaling
}

\author{
Dongwon Baek ${ }^{1,3}$, Hyun Jin Chun ${ }^{1,3}$, Songhwa Kang1, Gilok Shin', Su Jung Park', Hyewon Hong', \\ Chanmin Kim, Doh Hoon Kim², Sang Yeol Lee', Min Chul Kim ${ }^{1, *}$, and Dae-Jin Yun ${ }^{1, *}$
}

\begin{abstract}
MiR399f plays a crucial role in maintaining phosphate homeostasis in Arabidopsis thaliana. Under phosphate starvation conditions, AtMYB2, which plays a role in plant salt and drought stress responses, directly regulates the expression of miR399f. In this study, we found that miR399f also participates in plant responses to abscisic acid (ABA), and to abiotic stresses including salt and drought. Salt and ABA treatment induced the expression of miR399f, as confirmed by histochemical analysis of promoter-GUS fusions. Transgenic Arabidopsis plants overexpressing miR399f (miR399f-OE) exhibited enhanced tolerance to salt stress and exogenous $A B A$, but hypersensitivity to drought. Our in silico analysis identified ABF3 and CSP41b as putative target genes of miR399f, and expression analysis revealed that mRNA levels of ABF3 and CSP41b decreased remarkably in miR399f-OE plants under salt stress and in response to treatment with ABA. Moreover, we showed that activation of stress-responsive gene expression in response to salt stress and ABA treatment was impaired in miR399f-OE plants. Thus, these results suggested that in addition to phosphate starvation signaling, miR399f might also modulates plant responses to salt, $A B A$, and drought, by regulating the expression of newly discovered target genes such as ABF3 and CSP41b.
\end{abstract}

\section{INTRODUCTION}

During growth and development, plants encounter a wide array of environmental stresses that trigger physiological and genetic responses (Chinnusamy and Zhu, 2009; Cushman and Bohnert, 2000). In addition, primary stresses lead to secondary stresses such as oxidative stress and thermal shock. Accordingly, plants have evolved various response mechanisms that help them adapt or acclimate to the stresses (Yamaguchi-

${ }^{1}$ Division of Applied Life Science (BK21 Plus Program), Plant Molecular Biology and Biotechnology Research Center, Gyeongsang National University, Jinju 660-701, Korea, ${ }^{2}$ College of Life Science and Natural Resources, Dong-A University, Busan 604-714, Korea, ${ }^{3}$ These authors contributed equally to this work.

*Correspondence: djyun@gnu.ac.kr (DJY); mckim@gnu.ac.kr (MCK)

Received 2 July, 2015; revised 29 October, 2015; accepted 2 November, 2015; published online 15 December, 2015

Keywords: ABA, abiotic stress, arabidopsis, drought, microRNA, salt
Shinozaki and Shinozaki, 2006). A large proportion of plant genes are regulated by biotic (e.g., bacterial pathogens, virus, fungi, insects, and nematodes) (Brotman et al., 2012; Fagard et al., 2007) and abiotic stresses (e.g., drought, soil salinity, extreme temperatures, and heavy metals) (Chao et al., 2005; Si et al., 2009). Various cellular processes, such as RNA processing and post-transcriptional or even post-translational modifications, participate in regulation of the expression of genes in response to biotic and abiotic stresses.

MicroRNAs (miRNAs) can repress gene expression at the post-transcriptional level in plants (Bartel, 2004; Mallory and Vaucheret, 2006). MiRNAs are generally generated via a multistep process associated with the activation of DCL1 (DICERLIKE 1), HEN1, and HYL1 (Jones-Rhoades et al., 2006). The binding of miRNAs to the mRNAs of target genes leads to the degradation and/or translational inhibition of the targets (Guo et al., 2005; Voinnet, 2009).

Recent studies have shown that plants respond to environmental stresses by modulating gene expression at posttranscriptional levels via miRNAs. MiRNAs act in a wide variety of metabolic and biological processes during plant hormone signaling (Liu and Chen, 2009), abiotic stress responses (Lu and Huang, 2008; Sunkar et al., 2007), and immune responses (Katiyar-Agarwal and Jin, 2010; Lu et al., 2008; Voinnet, 2008). Similarly, numerous studies have revealed the involvement of specific miRNAs in plant responses to biotic and abiotic stresses. For example, the expression of miR169 is induced in response to drought (Li et al., 2008), cold (Zhou et al., 2008), salt (Zhao et al., 2009), nitrogen deficiency (Zhao et al., 2011), and UV-B radiation (Zhou et al., 2007). MiR393 is involved in nitrate signaling (Vidal et al., 2010), drought stress, and auxin signaling (Chen and Xiong, 2012). The expression of miR398 is suppressed in response to salt stress, abscisic acid (ABA) treatment (Jia et al., 2009), oxidative stress (Sunkar et al., 2006), high light (Siré et al., 2009), and biotic stress (Jagadeeswaran et al., 2009; Naya et al., 2014). MiR394 is involved in the regulation of plant responses to salt and drought stresses (Song et al., 2013). MiR395 is highly expressed in response to sulfate deficiency (Liang et al., 2010) as well as salt and dehydration (Kim et al., 2010). MiR159, miR397, and miR402 are up-regulated, but miR389 is down-regulated, in response to ABA (Reyes and Chua, 2007; Sunkar and Zhu, 2004). MiR399 expression is strongly induced by phosphate deficiency, and regulates phosphate uptake and root-to-shoot phosphate translocation as a systemic signaling molecule (Bari et al., 2006; Fujii et al., 2005; Pant et al., 2008). MiR156, miR778, miR827, and 
miR2111 are highly expressed under phosphate-deficiency conditions, whereas miR169, miR395, and miR398 are repressed by phosphate deficiency (Buhtz et al., 2008; Hsieh et al., 2009; Pant et al., 2009). Numerous studies have tested whether miRNAs function as linkers between nutrient homeostasis and hormone signaling or abiotic stress.

Previously we reported that miR399f, which acts in phosphate homeostasis, is directly activated by AtMYB2 (Baek et al., 2013). AtMYB2 regulates the expression of genes that respond to salt and drought stresses (Abe et al., 2003; Yoo et al., 2005). In this study, we revealed that miR399f plays an important role in plant responses to abiotic stresses, including salt and drought stresses. The transcription of miR399f is up-regulated by salt stress and exogenous ABA. Furthermore, we showed that transgenic Arabidopsis overexpressing miR399f displayed tolerance to salt stress and ABA treatment, but were hypersensitive to drought. Moreover, we identified candidate target genes of miR399f that function in plant abiotic stress signaling. Our study reports a novel biological function of miR399f and its possible regulatory mechanism in plant responses to abiotic stresses.

\section{MATERIALS AND METHODS}

\section{Plant materials and stress treatments}

Seedlings of Arabidopsis thaliana ecotype Colombia (Col-0) plant were sown and grown on 1/2 Murashige and Skoog (MS) medium containing $1.5 \%$ sucrose, and $0.6 \%$ agar, $\mathrm{pH} 5.7$ for 10 days, and then were treated with different stresses for indicated times (Figs. 1 and 3). Plants were grown in a growth chamber with a cycle of $16 \mathrm{~h}$ light (approximately $100 \mu \mathrm{E} \mathrm{m}^{-2} \mathrm{~s}^{-1}$ ) and $8 \mathrm{~h}$ dark at $22^{\circ} \mathrm{C}$.

Dr. Zhu kindly provided the seeds of wild type (Col-gl) and transgenic plants overexpressing miR399f (Fujii et al., 2005). These plants were grown on $1 / 2 \mathrm{MS}$ medium containing $1.5 \%$ sucrose, and $1.2 \%$ agar, $\mathrm{pH} 5.7$ for 4 days, and then transferred to media containing various concentrations of $\mathrm{NaCl}(0$ to $150 \mathrm{mM}$ ) and further grown for 8 days. To test cotyledon greening under $A B A$ treatment conditions, seeds of these plants were grown on $1 / 2 \mathrm{MS}$ medium without or with $\mathrm{ABA}(0$ to $1 \mu \mathrm{M})$ for 5 days.

For drought treatments, 3-week-old plants were treated with natural drought (water was withheld). After 12 days without watering, the drought-treated plants were re-watered, and recovery was checked after 1 day. Drought experiments were repeated five times and at least 7 plants for each individual line were used in each repeated experiment and one representative picture was shown.

\section{Measurement of water loss}

The rate of water loss by the leaves was measured. The shoots of 4-week-old plants were detached from the root, and weighed immediately. The shoots were placed in a covered plate at room temperature and weighed at various time intervals. The loss of fresh weight was calculated on the basis of the initial weight of the plant. At least three biological replicates for each sample were used for water loss assays.

\section{Small RNA and RNA gel blot analyses}

RNA was extracted from seedlings using Plant RNA Reagent (Invitrogen, USA) following the supplier's instructions. For detection of miR399f, $20 \mu \mathrm{g}$ total RNA was resolved on $15 \%$ polyacrylamide gels containing $7 \mathrm{M}$ urea and transferred electrophoretically to nylon membranes (EMD Millipore, USA) using semi-dry transfer (GE Healthcare, USA). Probes complementa- ry to miR399f were 5'-end labeled with $\gamma^{32} \mathrm{P}$-ATP using Optikinase (Usb, USA). For small RNA blotting, blots were prehybridized for at least $1 \mathrm{~h}$ and hybridized for $24 \mathrm{~h}$ using Perfecthyb Plus Hybridization Buffer (Sigma, USA) at $37^{\circ} \mathrm{C}$.

For detection of AtMYB2 mRNA, $15 \mu \mathrm{g}$ total RNA was separated on formaldehyde agarose gels and transferred to nylon membranes (EMD Millipore, USA). For hybridization, blots were pre-hybridized for at least $1 \mathrm{~h}$ and hybridized for $18 \mathrm{~h}$ at $65^{\circ} \mathrm{C}$. Blots were washed three times $(2 \times$ SSC and $0.1 \%$ SDS for 20 min, $0.5 \times$ SSC and $0.1 \%$ SDS for $20 \mathrm{~min}, 0.1 \times$ SSC and $0.1 \%$ SDS for $20 \mathrm{~min}$ ) at $50^{\circ} \mathrm{C}$. Ethidium bromide staining was used for RNA loading controls. The relative intensity of detected bands was measured with Image J program.

\section{RT-PCR and quantitative Real-time PCR analysis}

For RT-PCR, total RNA was isolated using an RNaeasy Kit (Qiagen, USA) according to the manufacturer's instructions. Total RNA was treated with DNase I (Qiagen, USA) to remove genomic DNA contamination. The first-strand cDNA was synthesized using $2 \mu \mathrm{g}$ total RNA with a cDNA synthesis kit (Invitrogen, USA), and subjected to RT-PCR analysis for examination of gene expression.

Quantitative real-time PCR (qRT-PCR) was used to assay gene expression levels with a CFX384TM Real-Time PCR Detection System (Bio-Rad, USA) following a standard protocol. The QuantiSpeed SYBR kit (PhileKorea, Korea) was used for $20 \mu \mathrm{l} \mathrm{PCR}$ reactions as follows: $50^{\circ} \mathrm{C}$ for $2 \mathrm{~min}, 95^{\circ} \mathrm{C}$ for $2 \mathrm{~min}$, and 40 cycles of $95^{\circ} \mathrm{C}$ for $5 \mathrm{~s}$ and $60^{\circ} \mathrm{C}$ for $30 \mathrm{~s}$. The relative expression levels of all samples were automatically calculated and analyzed three times by CFX Manager software (Bio-Rad, USA). The specific primers used in RT-PCR and qRT-PCR analysis are described in Supplementary Table 2. TUBULIN2 primers were used for RNA normalization.

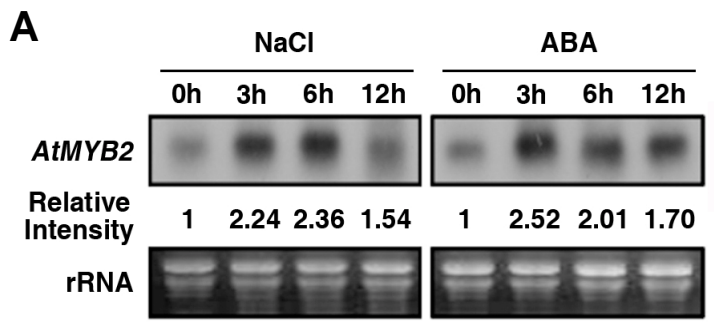

\section{B}

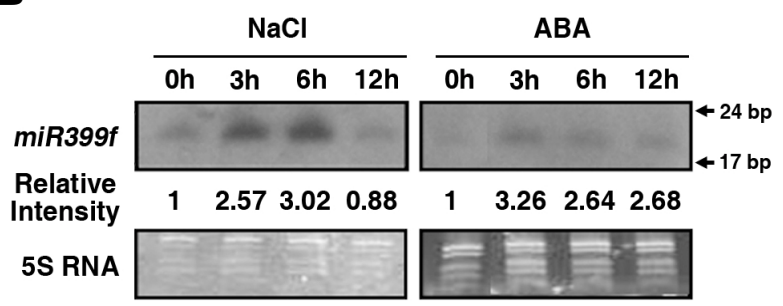

Fig. 1. Expression of $A t M Y B 2$ and miR399f under salt stress and ABA treatment. For RNA gel blot and small RNA blot analysis, total RNAs were extracted from ten-day-old seedlings treated with 100 $\mathrm{mM} \mathrm{NaCl}$ or $100 \mu \mathrm{M}$ ABA for the indicated times. (A) RNA gel blot analysis of AtMYB2 expression. (B) Small RNA blot analysis of miR399f expression. The $r R N A$ and $5 S$ RNA are shown as loading controls. "Relative Intensity" indicates the signal intensity relative to that at time 0 of treatment. 


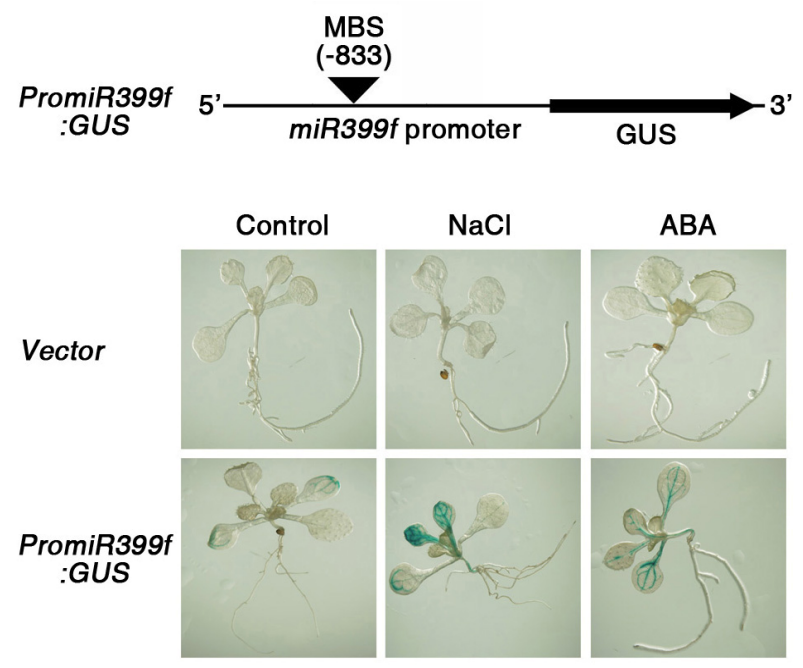

Fig. 2. Promoter activity of miR399f under salt and ABA treatment. Histochemical analysis of GUS activity was conducted using tenday-old PromiR399f:GUS and vector control transgenic seedlings treated with $100 \mathrm{mM} \mathrm{NaCl}$ or $100 \mu \mathrm{M} \mathrm{ABA}$ for $3 \mathrm{~h}$.

\section{Histological staining of GUS activity}

Transgenic plants of PromiR399f:GUS or vector-only controls (Baek et al., 2013) were grown on 1/2 MS medium for 10 days, and then treated $100 \mathrm{mM} \mathrm{NaCl}$ and $100 \mu \mathrm{M} \mathrm{ABA}$ for $3 \mathrm{~h}$. For GUS histological staining, seedlings from treated transgenic plants were incubated at $37^{\circ} \mathrm{C}$ for $6 \mathrm{~h}$ in the dark, in staining buffer $(0.5 \mathrm{M}$ Tris, $\mathrm{pH} 7.0,10 \%$ Triton X-100) with $1 \mathrm{mM}$ X-Gluc (5-bromo-4-chloro-3-indolyl $\beta$-D-glucuronide; Park et al., 2013). Chlorophyll was removed using an ethanol series: $20 \%, 35 \%$, and $50 \%$ ethanol at room temperature for $30 \mathrm{~min}$ each.

\section{In silico analysis}

Plant microRNA database (PMRD; http://bioinformatics.cau.edu. cn/PMRD/) and Web microRNA designer (WMD3; http://wmd3. weigelworld.org/cgi-bin/webapp.cgi) were used to search for putative target genes of miR399f.

\section{RESULTS}

Expression of AtMYB2 and miR399f in response to salt stress and ABA treatment

Previously we showed that $A t M Y B 2$, a transcription factor that functions in ABA and salt stress signaling in Arabidopsis, also acts in phosphate starvation signaling by regulating miR399f transcription (Baek et al., 2013; Fujii et al., 2005). To test whether miR399f participates in plant responses to ABA and salt stress signaling, we analyzed patterns of AtMYB2 and miR399f expression in ten-day-old Arabidopsis seedlings treated with $100 \mathrm{mM} \mathrm{NaCl}$ and $100 \mu \mathrm{M} \mathrm{ABA}$ for various times (Fig. 1). Consistent with previous reports (Urao et al., 1993), the expression of AtMYB2 increased strongly in response to $\mathrm{NaCl}$ and $\mathrm{ABA}$ treatment (Fig. 1A). The transcript level of $A t-$ MYB2 increased within $3 \mathrm{~h}$ of $\mathrm{NaCl}$ and $\mathrm{ABA}$ treatment and stayed high up to $6 \mathrm{~h}$ of treatment. Similarly, miR399f was strongly induced by $100 \mathrm{mM} \mathrm{NaCl}$ or $100 \mu \mathrm{M}$ ABA (Fig. 1B). Thus, miR399f and AtMYB2 showed similar expression patterns. These results suggested that miR399f, which plays an

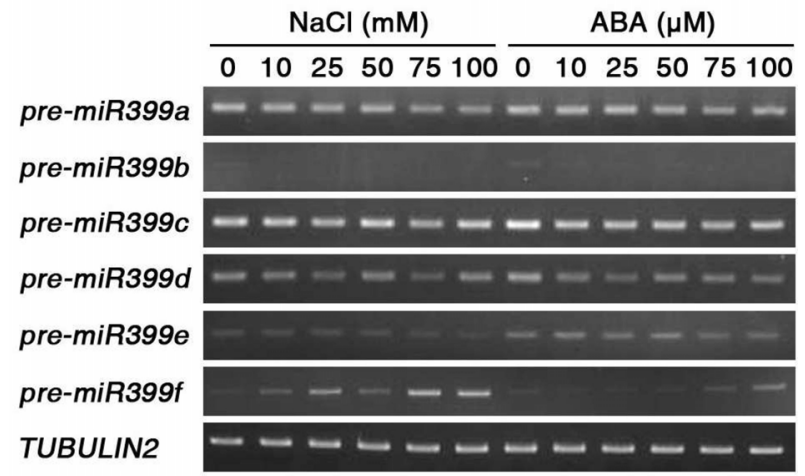

Fig. 3. Expression of precursors of miR399 family members in response to salt stress and ABA treatment. Total RNA extracted from ten-day-old seedlings after treatment with different concentrations of $100 \mathrm{mM} \mathrm{NaCl}$ or $100 \mu \mathrm{M} \mathrm{ABA}$ for $3 \mathrm{~h}$. TUBULIN2 was used as a loading control.

important role in phosphate homeostasis, might also participate in salt stress and $A B A$ signaling and moreover that the regulation of miR399f expression during salt stress and ABA treatment involves AtMYB2.

To further investigate the regulation of miR399f expression by salt and $A B A$ treatment, we performed histochemical staining for $\beta$-glucuronidase (GUS) expression in one-week-old transgenic Arabidopsis plants harboring the miR399f promoter fused to the GUS reporter gene (PromiR399f:GUS) and grown in media containing $\mathrm{NaCl}$ or ABA (Fig. 2). Transgenic plants harboring the empty GUS vector were used as a control. Strong promoter activity of miR399f was detected in vascular tissues of rosette leaves of seedlings grown in the presence of salt or ABA, whereas very weak GUS staining was observed in PromiR399f:GUS seedlings grown under normal conditions. GUS expression in PromiR399:GUS plants was mainly observed in vascular tissues under salt and ABA stress condition, but we could also detected weaker expression of GUS in leaf tissues (Supplementary Fig. S1). It is possible that miR399f generated from vascular tissue can move to neighboring leaf tissues and regulate its target gene expression, because cell-to-cell movement of miRNA has been previously reported (Lin et al., 2008). These results confirmed that miR399f expression was influenced by salt and ABA.

\section{Expression of miR399 family members in response to salt and ABA treatments}

In Arabidopsis thaliana, the miR399 family consists of six members, miR399a to miR399f. Pi starvation conditions induce the expression of miR399 family members, which regulate the expression of an ubiquitin-conjugating enzyme 24 (UBC24) gene involved in phosphate homeostasis (Aung et al., 2006). To investigate the responses of the miR399 family members to salt and ABA stresses, we analyzed transcript levels of miR399 family genes by RT-PCR in ten-day-old seedlings that had been treated with different concentrations of $\mathrm{NaCl}$ and $\mathrm{ABA}$ for $3 \mathrm{~h}$ (Fig. 3). With increasing concentrations of $\mathrm{NaCl}$ and $\mathrm{ABA}$, the expression of the miR399f precursor remarkably increased. In contrast to miR399f, $\mathrm{NaCl}$ and ABA treatments did not affect the expression of other miR399 family members, such as miR399a, miR399b, miR399c, miR399d, and miR399e. This 
A
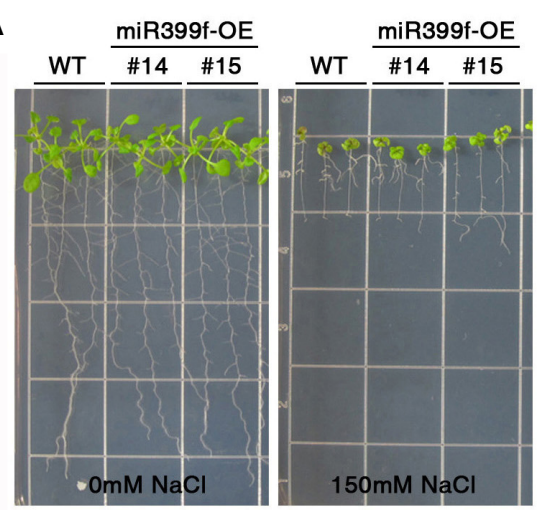

C

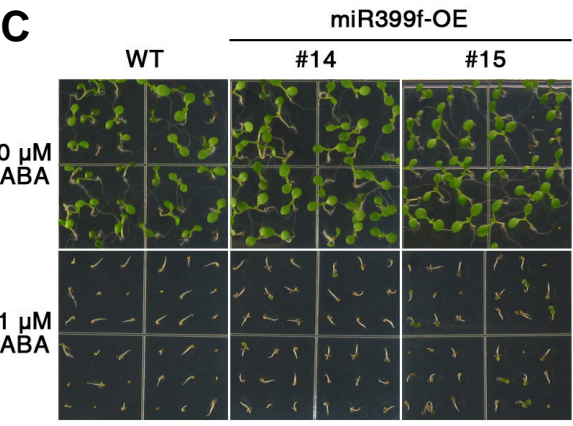

E

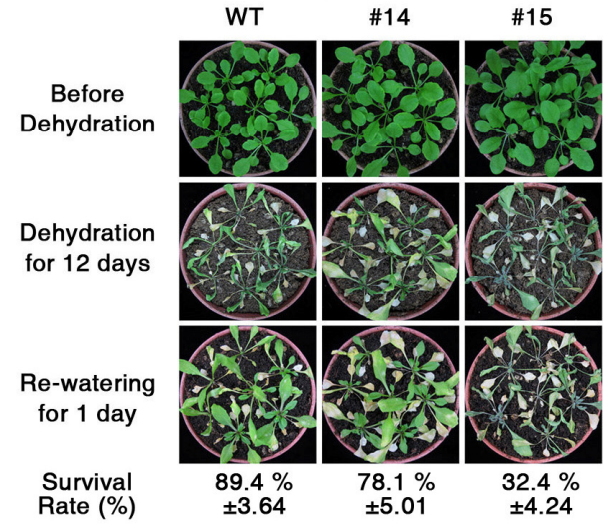

B

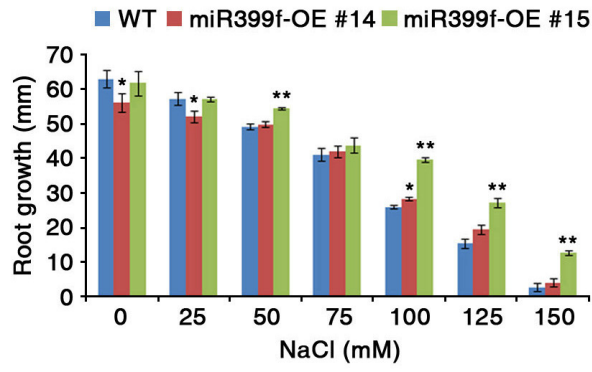

D

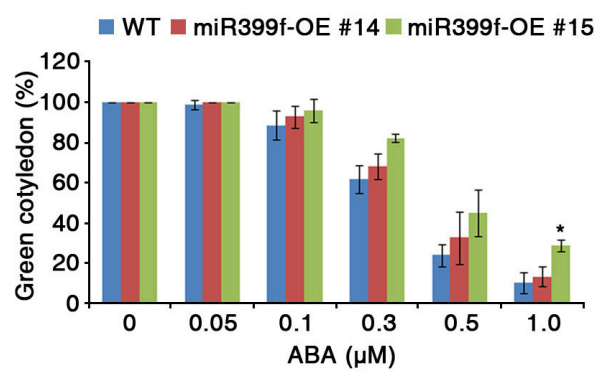

$\boldsymbol{F}$

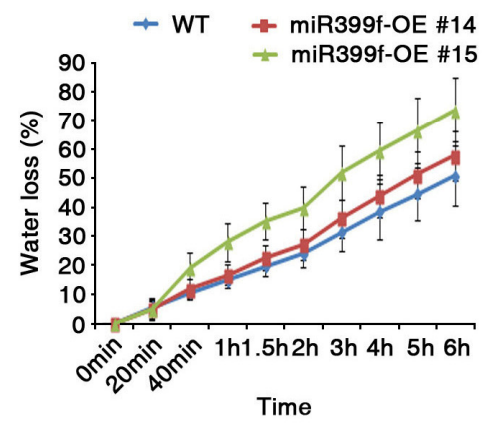

Fig. 4. Responses of miR399foverexpressing plants to salt, $A B A$ and drought. (A) WT and two independent lines overexpressing miR399f (miR399f-OE \#14 and \#15) were grown on $1 / 2 \mathrm{MS}$ agar medium for 4 days, and then transferred to $1 / 2 \mathrm{MS}$ agar without $\mathrm{NaCl}$ $(0 \mathrm{mM} \mathrm{NaCl})$ or with $\mathrm{NaCl}(150 \mathrm{mM}$ $\mathrm{NaCl}$ ), and incubated for 8 days. (B) Comparison of root elongation at different concentrations of $\mathrm{NaCl}$ in WT and miR399f-OE plants. Bars represent the means \pm standard error of three replicates with 16 seedlings per replicate. Asterisks represent significant differences from the WT $\left(^{*} ; p\right.$-value $\leq 0.05$, Student's $t$-test). (C) Seeds of WT and miR399f-OE plants were germinated on $1 / 2 \mathrm{MS}$ agar medium without $\mathrm{ABA}(0 \mu \mathrm{M}$ ABA) or with ABA (1 $\mu \mathrm{M}$ ABA) for 5 days. (D) Comparison of cotyledon greening at different concentrations of ABA in WT and miR399-OE plants. Bars represent the means \pm standard error of three replicates with 30 seeds per replicate. Asterisks represent significant differences from the WT (*; p-value $\leq 0.05$, Student's $t$-test). (E) Photographs show plants before and after dehydration stress. WT and miR399f-OE plants were grown in soil with sufficient water for 3 weeks, and then the water were withheld for 12 days. Plants were re-watered for 1 day before the photograph was taken. (F) Water loss from detached leaves of 4-week-old plants measured at room temperature. Bars represent the means \pm standard error of three replicates with 5 seedlings per replicate. result suggested that the expression of miR399f, but not other miR399 family members, is induced by not only $\mathrm{Pi}$ starvation, but also by $\mathrm{NaCl}$ stress and $\mathrm{ABA}$.

\section{miR399f-overexpressing plants have different responses to $\mathrm{NaCl}, \mathrm{ABA}$, and drought}

We obtained two Arabidopsis transgenic lines overexpressing miR399f (kindly provided by Dr. Jian-Kang Zhu); one line showed relatively weak expression of miR399f (\#14) and the other line showed strong expression (\#15) (Fujii et al., 2005; Supplementary Fig. S2). To determine the effect of miR399f overexpression on plant responses to salt stress, we performed root growth assays on Murashige and Skoog (MS) medium containing various concentrations of $\mathrm{NaCl}$ (Figs. 4A and 4B). Four-day-old seedlings of wild type (WT) and miR399foverexpressing plants (miR399f-OE \#14 and \#15) grown on
MS medium were transferred to MS medium containing different concentrations of $\mathrm{NaCl}$ and their primary root lengths were measured. The primary root elongation of WT seedlings was strongly suppressed under $100 \mathrm{mM} \mathrm{NaCl}$ conditions, in which root length was approximately $41 \%$ that of WT seedlings grown under normal conditions. However, the root elongation of miR399f-OE \#15 seedlings showed more resistance to $\mathrm{NaCl}$ treatment. At 100,125 , and $150 \mathrm{mM} \mathrm{NaCl}$, the root length of miR399f-OE \#15 seedlings was 1.52-, 1.75-, and 4.83-fold that of WT plants grown under the same conditions, respectively (Fig. 4B). By contrast, the root elongation of miR399f-OE \#14 was similar to that of the WT plants under $\mathrm{NaCl}$ stress conditions.

We further examined the response of miR399f-OE plants to ABA treatment by measuring the number of green cotyledons after seed germination (Figs. 4C and 4D). The seeds of WT 
A
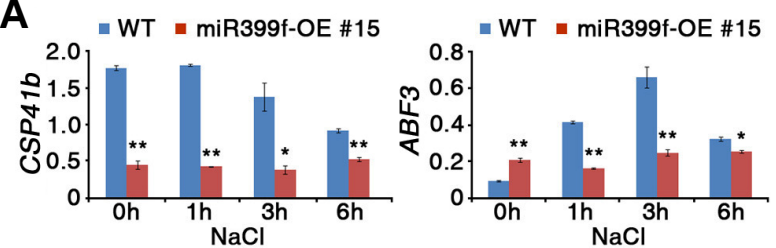

B
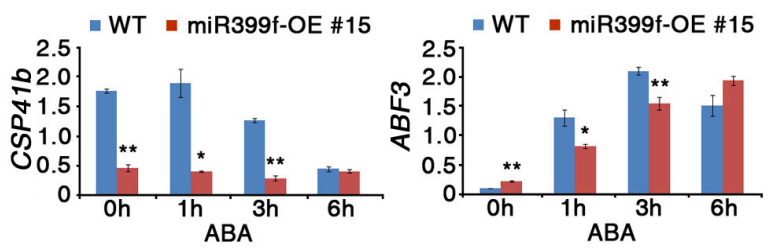

C
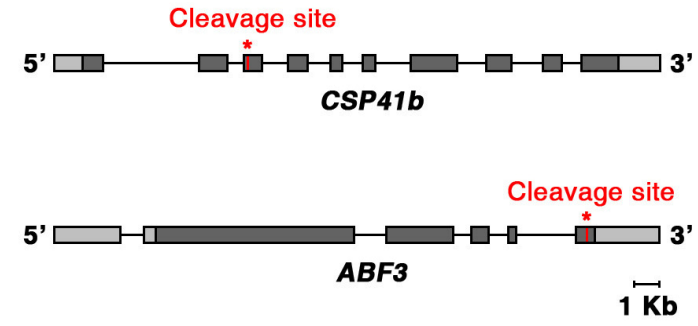

口 Exon a UTR - Intron

Fig. 5. Expression of putative target genes of miR399f. (A, B) Analysis by qRT-PCR of mRNA levels of CSP41b and ABF3 in ten-dayold WT and miR399f-OE seedlings grown in the presence of $\mathrm{NaCl}$ (A) or ABA (B). Total RNA was extracted from ten-day-old seedlings after $100 \mathrm{mM} \mathrm{NaCl}$ or $100 \mu \mathrm{M}$ ABA treatment for the indicated times. TUBULIN2 was used for normalization. Bars represent the means \pm standard error of three biological replicates with two technical replicates each. Asterisks represent significant differences from the WT ( ${ }^{*}$; $0.01<\mathrm{p}$-value $\leq 0.05,{ }^{* *}$; p-value $\leq 0.01$, Student's $t$-test). (C) Schematic diagram of the CSP $41 b$ and $A B F 3$ genes. Red asterisks indicate the predicted cleavage sites of miR399f.

and miR399f-OE plants were germinated on MS media containing 0 to $1 \mu \mathrm{M} \mathrm{ABA}$ and grown for 5 days. The seeds of WT and miR399f-OE plants showed different responses to ABA treatment. At $0.3 \mu \mathrm{M}$ ABA, approximately $82 \%$ of miR399f-OE \#15 seedlings developed green cotyledons, compared to approximately $62 \%$ of WT seedlings. The difference was more obvious at higher concentrations of ABA. At $1 \mu \mathrm{M} A B A$, the number of miR399f-OE \#15 seedlings with green cotyledons was 2.78 -fold higher than that of WT seedlings.

In contrast to their tolerance of $\mathrm{NaCl}$ and $\mathrm{ABA}$, miR399f-OE plants were more sensitive to drought than WT plants. Threeweek-old WT and miR399f-OE plants were subjected to drought stress for 12 days and then re-watered. Under drought conditions, most WT and miR399f-OE plants withered, but one day after re-watering, WT plants resumed growth, whereas miR399f-OE plants had not fully recovered (Fig. 4E). To further confirm the response to drought stress, we examined water loss of WT and miR399f-OE plants, using detached rosette leaves of 4-week-old plants and placing them on petri dishes at room temperature. Water loss proceeded more quickly from leaves of miR399f-OE plants than from WT leaves (Fig. 4F). At $6 \mathrm{~h}$ after detachment, the leaves of miR399f-OE plants lost almost $74 \%$ of their water, while WT leaves lost only $51 \%$. To- gether, these results indicated that miR399f-overexpressing plants were resistant to $\mathrm{NaCl}$ and $\mathrm{ABA}$, but hypersensitive to drought stress. Moreover, these findings support the idea that miR399f plays a role in plant responses to multiple abiotic stresses.

CSP41b and ABF3 are putative target genes of miR399f in salt stress and ABA signaling

The post-transcriptional activity of UBC24 is partially inhibited upon an increase in miR399f expression during the phosphatedeficiency response (Fujii et al., 2005). To understand the mode of action of miR399f in plant responses to salt, ABA, and drought, we attempted to identify the putative target genes of miR399f by in silico analysis using the Plant MicroRNA Database (PMRD) and Web MicroRNA Designer (WMD3) (Supplementary Table 1). In silico analysis revealed five putative miR399f target genes, BASS2 (BILE ACID:SODIUM SYMPORTER FAMILY PROTEIN 2), CSP41b, ABF3 (ABA-RESPONSIVE ELEMENT-BINDING TRANSCRIPTION FACTOR3), At1g04985, and At3g26730, in addition to UBC24. If miR399f exerts its role by mRNA cleavage, levels of the putative target mRNA should decrease in the miR399f-overexpressing plants. To determine whether miR399f mediates the mRNA cleavage of these target genes, we analyzed the transcript levels of the five putative target genes by quantitative real time-PCR (qRT-PCR) in WT and miR399f-OE \#15 plants grown in the presence of $\mathrm{NaCl}$ and $\mathrm{ABA}$ (Fig. 5A and $B$, Supplementary Fig. S3). We performed this study with miR399f-OE \#15 line, because miR399f-OE \#15 plants expressed more miR399f than miR399f-OE \#14 line and both lines showed similar phenotype under stress conditions (Fig. 4). Indeed, two candidate target genes, CSP41 b and ABF3, were down-regulated in miR399f-OE plants under salt stress conditions. In addition, the mRNA level of CSP41b decreased in miR399f-OE plants in the presence of ABA. However, the other three putative target genes (BASS2, At1g04985, and At3g26730) showed no significant difference in mRNA levels between WT and miR399f-OE plants (Supplementary Fig. S3). $A B F 3$ is involved in salt stress, $A B A$, and drought stress signaling and regulates the expression of abiotic stress-responsive genes (Finkelstein et al., 2005; Yoshida et al., 2010). CSP41b encodes a chloroplast RNA binding protein and to date has not been reported to be involved in abiotic stress. The qRT-PCR results showed that the expression of $C S P 41 b$ gradually decreased in response to $\mathrm{NaCl}$ and $\mathrm{ABA}$ treatment in WT plants, suggesting a possible role for CSP41b in stress signaling (Figs. $5 \mathrm{~A}$ and $5 \mathrm{~B}$ ). In silico analysis suggested that sites for cleavage by miR399f are located at the 3rd exon of CSP41b and 5th exon of $A B F 3$ (Fig. $5 \mathrm{C}$ ), although there are five mismatches, $G$ $A, G-G, G-U, A-A$ and $U-C$, or three mismatches, $C-U, G-G$ and $C-U$, between miR399f and the CSP41b and ABF3 mRNAs, respectively (Supplementary Table 1 ). Our results suggested that $C S P 41 \mathrm{~b}$ and $A B F 3$ are candidate target genes regulated by miR399f during salt stress and/or ABA signaling. Moreover, the results also suggested that the regulatory mechanism of ABF3 expression might be different in $\mathrm{NaCl}$ - and $\mathrm{ABA}$ responsive signaling pathways.

\section{Expression of stress-responsive genes in} miR399f-overexpressing plants in response to $\mathrm{NaCl}$ or ABA treatment

The expression of stress-responsive genes is remarkably impaired in the abf3 mutant (Yoshida et al., 2010). The downregulation of $A B F 3$ expression in miR399f-OE plants prompted us to test the expression of stress-responsive genes in Arabidopsis transgenic plants overexpressing miR399f. To investigate 
A
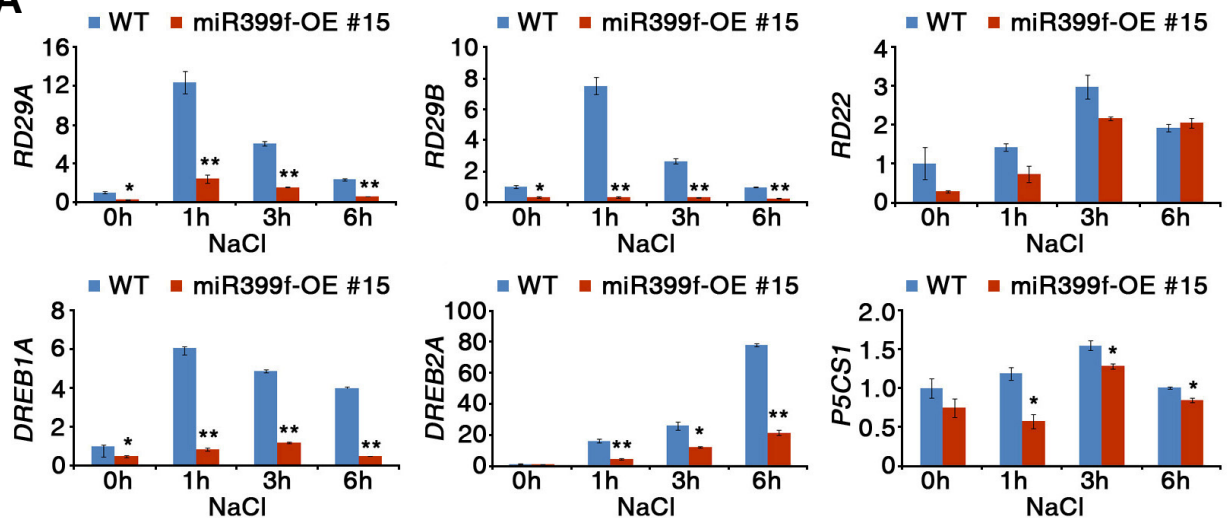

B
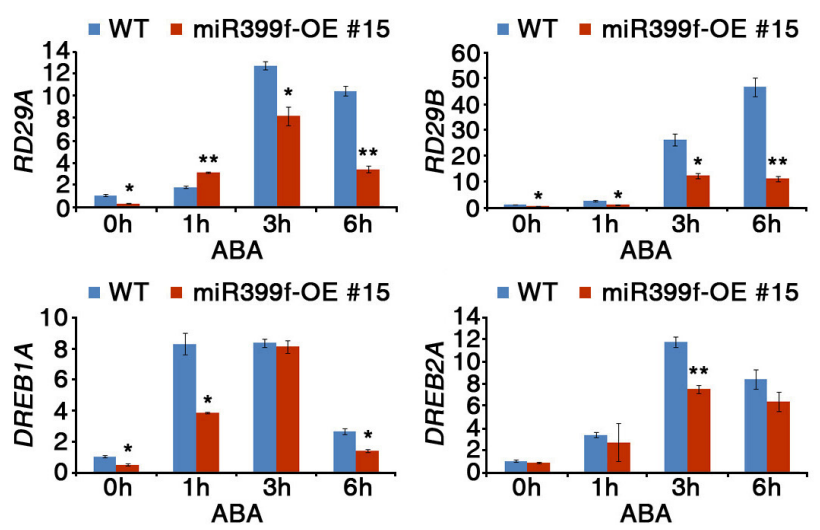

whether overexpression of miR399f affects the expression of stress-responsive genes, we analyzed the mRNA levels of stress-responsive genes such as $R D 29 A, R D 29 B, R D 22$, DREB1A, DREB2A, and P5CS1 in WT and miR399f-OE \#15 plants treated with $\mathrm{NaCl}$ or ABA. In WT plants, expression of these genes was highly induced by treatment with $\mathrm{NaCl}$ or $\mathrm{ABA}$ (Fig. 6). In miR399f-OE \#15 plants, however, induction of stress-responsive gene expression by $\mathrm{NaCl}$ and $\mathrm{ABA}$ treatments was significantly suppressed. This result was consistent with the observations in abf3 mutants (Yoshida et al., 2010). The suppression was more obvious for $R D 29 A, R D 29 B$, $D R E B 1 A$, and $D R E B 2 A$ expression in response to $\mathrm{NaCl}$ treatment (Fig. 6A), and for $R D 29 B$ and $R D 22$ in response to exogenous ABA (Fig. 6B). These results suggested that miR399f contributes to the regulation of stress-responsive gene expression in response to salt, $A B A$, and drought. Taken together, our results suggested the existence of a novel regulatory mechanism mediated by miR399f in plant responses to abiotic stresses.

\section{DISCUSSION}

Abiotic stresses affect various physiological processes in plant development, such as seedling growth and seed germination. Exposure to different abiotic stresses can lead to similar responses in plants. Moreover, different kinds of stresses can trigger responses through the induction of similar types of miRNAs (Sunkar and Zhu, 2004). This suggests that plants share common signaling pathways that act in different abiotic stress responses. The relevant miRNAs are either up- or down-regulated
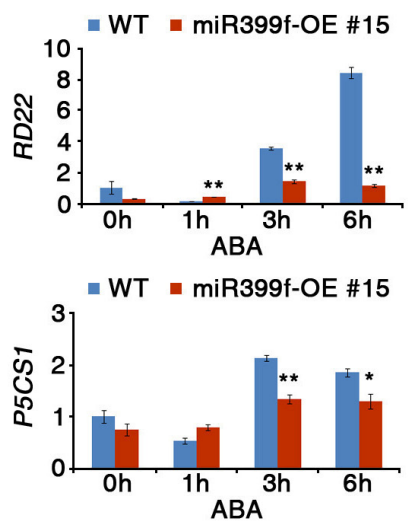

Fig. 6. Expression of stressresponsive genes in miR399f$\mathrm{OE}$ plants in response to salt stress and ABA. The mRNA levels of stress-responsive genes in WT and miR399f-OE in the presence of $\mathrm{NaCl}(\mathrm{A})$ or ABA (B) were determined by qRT-PCR using total RNA extracted from ten-day-old seedlings after treatment with 100 $\mathrm{mM} \mathrm{NaCl}$ or $100 \mu \mathrm{M}$ ABA for the indicated times. TUBULIN2 was used for normalization. Bars represent the means \pm standard error of three biological replicates with two technical replicates each. Asterisks represent significant differences from the WT $\left(* ; 0.01<p\right.$-value $\leq 0.05,{ }^{* *}$; $\mathrm{p}$-value $\leq 0.01$, Student's $t$-test). after exposure to stress treatments that influence plant growth and developmental processes (Lu and Huang, 2008). The role of miR399 has been established mainly in the plant response to phosphate starvation (Bari et al., 2006). However, little is known about the potential biological function of miR399 in responses to other abiotic stresses. In this report, we uncovered a possible role of miR399f in plant responses to osmotic stresses, including salt, drought, and ABA. The miR399 family consists of six members, miR399a to miR399f, which share similar mature sequences. A previous report indicated that phosphate starvation induces the expression of miR399 family genes (Bari et al., 2006). Our data showed that the expression of miR399f was remarkably induced by $\mathrm{NaCl}$ and $\mathrm{ABA}$ treatment. These results suggested that the members of miR399 family might have distinct roles in plant responses to various abiotic stresses.

Several miRNAs function in the ABA-mediated stress response (Chen et al., 2012; Jia et al., 2009; Reyes and Chua, 2007). Some studies suggested a genetic connection between miRNAs and ABA-mediated stress responses (Kim et al., 2010; Song et al., 2013). Consistent with our results indicating the involvement of Arabidopsis miR399f in salt stress and ABA responses, miRNA array analysis revealed that poplar miR399 is induced in response to $\mathrm{ABA}$ and $\mathrm{NaCl}$ (Jia et al., 2009). These findings indicate that some stress-responsive miRNAs might be similarly regulated across different species. These miRNAs potentially play vital roles in the morphological and metabolic adaptation of plants to salinity and ABA-mediated stress responses, and a genotype-specific expression model might explain the distinct stress tolerances among species. 
Plant responses to various stresses are associated with multiple transcriptional cascades mediated by miRNAs (Lu and Huang, 2008; Sunkar et al., 2007). Identification of direct downstream target genes regulated by miRNAs in each of these cascades is crucial for understanding miRNA-mediated plant responses to stresses. Through our in silico prediction and subsequent gene expression analysis, we identified two candidate downstream target genes for miR399f, namely CSP41b and $A B F 3$, the expression levels of which significantly decreased in miR399f-OE plants under salt and ABA treatment conditions. However, the expression patterns of CSP41b and $A B F 3$ genes were different in WT under the stress conditions. Unlike CSP41b, ABF3 expression was decreased after $6 \mathrm{~h}$ of stress treatment. These results suggested that the mode of miR399f action on the regulation of CSP41b and ABF3 expression may be different. One possibility would be that miR399f regulate $A B F 3$ expression at the late stage to turn-off the $A B F 3$ mediated stress signaling. In the future, we are going to verify the specific regulatory mechanism for miR399f-mediated $C S P 41 b$ and $A B F 3$ gene expression.

While the role of CSP41b is not yet clear, it is known that $A B F 3$ is a transcription factor involved in transcriptional cascades in response to salt, $\mathrm{ABA}$ and drought. Mutation of $A B F 3$ enhances the tolerance of plant to salt stress and $A B A$ treatment, but significantly reduces their capacity to survive drought stress (Finkelstein et al., 2005; Yoshida et al., 2010). Consistent with the phenotype of the abf3 mutant under abiotic stresses, miR399f-OE plants displayed increased tolerance to salt stress and exogenous ABA, but hypersensitivity to drought stress (Fig. 4). These results indicate that miR399f acts as a positive regulator of plant tolerance of salt stress and $A B A$, but a negative regulator for plant response to drought stress. Recent work reported that miR168a- and miR394a-overexpressing plants were hypersensitive to salt stress, but resistant to drought stress (Li et al., 2012; Song et al., 2013). These results support our findings that same miRNA can have different, stressspecific roles in plant responses. Our findings point to a need to explore the role of $C S P 41 b$ and its regulation by miR399f for better understanding of the function of miR399f in plant stress signaling.

Overall, the present study identified a biological function of miR399f. Genetic and physiological studies revealed that overexpression of miR399f resulted in salt and ABA tolerance in Arabidopsis by overcoming the arrest of root growth and seed germination under salt and ABA treatment. By contrast, miR399f-OE plants displayed a hypersensitive phenotype under drought conditions (Fig. 4). In miR399f-OE plants, the expression of several stress-responsive genes, such as $R D 29 A$, $R D 29 B, D R E B 1 A$, and DREB2A was suppressed. Even though our results suggested that miR399f might function in abiotic stress signaling via $A B F 3$ and $C S P 41 b$, but still we cannot rule out the possibility of the involvement of other genes in miR399fmediated signaling. That's the reason why we tested a number of stress-responsive genes in our experiment. Moreover, we showed that $R D 29 B$ containing ABRE element in its promoter was down regulated in miR399f-OE plants (Fig. 6). This impaired induction of stress-responsive genes in miR399f-OE plant might contribute, at least in part, to its hypersensitive phenotype under drought conditions. Further characterization of the putative components might reveal the association between miR399f and salt stress or ABA signals.

In summary, we provide the first evidence of the involvement of miR399f in osmotic stress signaling, including responses to salt, $A B A$, and drought, and of its putative downstream target genes in Arabidopsis. Our results suggested that the regulation of $C S P 41 b$ and/or $A B F 3$ expression by miR399f would be important for the maintenance of a phenotype favorable for the adaptive responses to salt stress, ABA, and drought stresses. Furthermore, miR399f and its target genes have distinct roles in plant responses to different types of environmental stresses.

Note: Supplementary information is available on the Molecules and Cells website (www.molcells.org).

\section{ACKNOWLEDGMENTS}

This work was supported by a grant from National Research Foundation funded by the Gyeongsang National University Fund for Professors on Sabbatical Leave (2015), Korean Government (MSIP; 2013R1A2A1A01005170) and NextGeneration BioGreen21 Program (SSAC, grant\#: PJ01105101 and PJ01105102), Rural Development Administration Republic of Korea; and Basic Science Research Program through the National Research Foundation of Korea (NRF) funded by the Ministry of Education (NRF-2013R1A1A2011511) to D. Baek.

\section{REFERENCES}

Abe, H., Urao, T., Ito, T., Seki, M., Shinozaki, K., and YamaguchiShinozaki, K. (2003). Arabidopsis AtMYC2 (bHLH) and AtMYB2 (MYB) function as transcriptional activators in abscisic acid signaling. Plant Cell 15, 63-78.

Aung, K., Lin, S.I., Wu, C.C., Huang, Y.T., Su, C.L., and Chiou, T.J. (2006). pho2, a phosphate overaccumulator, is caused by a nonsense mutation in a microRNA399 target gene. Plant Physiol. 141, 1000-1011.

Baek, D., Kim, M.C., Chun, H.J., Kang, S., Park, H.C., Shin, G., Park, J., Shen, M., Hong, H., Kim, W.Y., et al. (2013). Regulation of miR399f transcription by AtMYB2 affects phosphate starvation responses in Arabidopsis. Plant Physiol. 161, 362-373.

Bari, R., Datt Pant, B., Stitt, M., and Scheible, W.R. (2006). PHO2, microRNA399, and PHR1 define a phosphate-signaling pathway in plants. Plant Physiol. 141, 988-999.

Bartel, D.P. (2004). MicroRNAs: genomics, biogenesis, mechanism, and function. Cell 116, 281-297.

Brotman, Y., Lisec, J., Méret, M., Chet, I., Willmitzer, L., and Viterbo, A. (2012). Transcript and metabolite analysis of the Trichoderma-induced systemic resistance response to Pseudomonas syringae in Arabidopsis thaliana. Microbiology 158, 139-146.

Buhtz, A., Springer, F., Chappell, L., Baulcombe, D.C., and Kehr, J. (2008). Identification and characterization of small RNAs from the phloem of Brassica napus. Plant J. 53, 739-749.

Chao, D.Y., Luo, Y.H., Shi, M., Luo, D., and Lin, H.X. (2005). Saltresponsive genes in rice revealed by cDNA microarray analysis. Cell Res. 15, 796-810.

Chen, H., Li, Z., and Xiong, L. (2012). A plant microRNA regulates the adaptation of roots to drought stress. FEBS Lett. 586, 17421747.

Chinnusamy, V., and Zhu, J.K. (2009). Epigenetic regulation of stress responses in plants. Curr. Opin. Plant Biol. 12, 133-139.

Cushman, J.C., and Bohnert, H.J. (2000). Genomic approaches to plant stress tolerance. Curr. Opin. Plant Biol. 3, 117-124.

Fagard, M., Dellagi, A., Roux, C., Périno, C., Rigault, M., Boucher, V., Shevchik, V.E., and Expert, D. (2007). Arabidopsis thaliana expresses multiple lines of defense to counterattack Erwinia chrysanthemi. Mol. Plant Microbe. Interact. 20, 794-805.

Finkelstein, R., Gampala, S.S., Lynch, T.J., Thomas, T.L., and Rock, C.D. (2005). Redundant and distinct functions of the ABA response loci ABA-INSENSITIVE(ABI) 5 and ABRE-BINDING FACTOR (ABF)3. Plant Mol. Biol. 59, 253-267.

Fujii, H., Chiou, T.J., Lin, S.I., Aung, K., and Zhu, J.K. (2005). A miRNA involved in phosphate-starvation response in Arabidopsis. Curr. Biol. 15, 2038-2043.

Guo, H.S., Xie, Q., Fei, J.F., and Chua, N.H. (2005). MicroRNA directs mRNA cleavage of the transcription factor NAC1 to downregulate auxin signals for arabidopsis lateral root development. Plant Cell 17, 1376-1386. 
Hsieh, L.C. Lin, S.I. Shih, A.C., Chen, J.W., Lin, W.Y, Tseng, C.Y. Li, W.H., and Chiou, T.J. (2009). Uncovering small RNAmediated responses to phosphate deficiency in Arabidopsis by deep sequencing. Plant Physiol. 151, 2120-2132.

Jagadeeswaran, G., Saini, A., and Sunkar, R. (2009). Biotic and abiotic stress down-regulate miR398 expression in Arabidopsis. Planta 229, 1009-1014.

Jia, X., Wang, W.X., Ren, L., Chen, Q.J., Mendu, V., Willcut, B., Dinkins, R., Tang, X., and Tang, G. (2009). Differential and dynamic regulation of miR398 in response to ABA and salt stress in Populus tremula and Arabidopsis thaliana. Plant Mol. Biol. 71, 51-59.

Jones-Rhoades, M.W., Bartel, D.P., and Bartel, B. (2006). MicroRNAS and their regulatory roles in plants. Annu. Rev. Plant Biol. 57, 19-53.

Katiyar-Agarwal, S., and Jin, H. (2010). Role of small RNAs in hostmicrobe interactions. Annu. Rev. Phytopathol. 48, 225-246.

Kim, J.Y., Lee, H.J., Jung, H.J., Maruyama, K., Suzuki, N., and Kang, H. (2010). Overexpression of microRNA395c or 395e affects differently the seed germination of Arabidopsis thaliana under stress conditions. Planta 232, 1447-1454

Li, W.X., Oono, Y., Zhu, J., He, X.J., Wu, J.M., lida, K., Lu, X.Y., Cui, X., Jin, H., and Zhu, J.K. (2008). The Arabidopsis NFYA5 transcription factor is regulated transcriptionally and posttranscriptionally to promote drought resistance. Plant Cell 20, 2238-2251.

Liang, G., Yang, F., and Yu, D. (2010). MicroRNA395 mediates regulation of sulfate accumulation and allocation in Arabidopsis thaliana. Plant J. 62, 1046-1057.

Lin, S.I., Chiang, S.F., Lin, W.Y., Chen, J.W., Tseng, C.Y., Wu, P.C., and Chiou, T.J. (2008). Regulatory network of microRNA399 and $\mathrm{PHO} 2$ by systemic signaling. Plant Physiol. 147, 732-746.

Liu, Q., and Chen, Y.Q. (2009). Insights into the mechanism of plant development: interactions of miRNAs pathway with phytohormone response. Biochem. Biophys. Res. Commun. 384, 1-5.

Lu, X.Y., and Huang, X.L. (2008). Plant miRNAs and abiotic stress responses. Biochem. Biophys. Res. Commun. 368, 458-462.

Lu, Y.D., Gan, Q.H., Chi, X.Y., and Qin, S. (2008). Roles of microRNA in plant defense and virus offense interaction. Plant Cell Rep. 27, 1571-1579.

Mallory, A.C., and Vaucheret, H. (2006). Functions of microRNAs and related small RNAs in plants. Nat. Genet. 38, S31-S36.

Naya, L., Paul, S., Valdés-López, O., Mendoza-Soto, A.B., NovaFranco, B., Sosa-Valencia, G., Reyes, J.L., and Hernández, G. (2014). Regulation of copper homeostasis and biotic interactions by microRNA 398b in common bean. PLoS One 9 , e84416.

Pant, B.D., Buhtz, A., Kehr, J., and Scheible, W.R. (2008). MicroRNA399 is a long-distance signal for the regulation of plant phosphate homeostasis. Plant J. 53, 731-738.

Pant, B.D., Musialak-Lange, M., Nuc, P., May, P., Buhtz, A., Kehr, J., Walther, D., and Scheible, W.R. (2009). Identification of nutrientresponsive Arabidopsis and rapeseed microRNAs by comprehensive real-time polymerase chain reaction profiling and small RNA sequencing. Plant Physiol. 150, 1541-1555

Park, M.Y., Kim, S.A., Lee, S.J., and Kim, S.Y. (2013). ATHB17 is a positive regulator of abscisic acid response during early seedling growth. Mol. Cells 35, 125-133.

Reyes, J.L., and Chua, N.H. (2007). ABA induction of miR159 controls transcript levels of two MYB factors during Arabidopsis seed germination. Plant J. 49, 592-606.

Si, Y., Zhang, C., Meng, S., and Dane, F. (2009). Gene expression changes in response to drought stress in Citrullus colocynthis. Plant Cell Rep. 28, 997-1009.

Siré, C., Moreno, A.B., Garcia-Chapa, M., López-Moya, J.J. and San Segundo, B. (2009). Diurnal oscillation in the accumulation of Arabidopsis microRNAs, miR167, miR168, miR171 and miR398. FEBS Lett. 583, 1039-1044.

Song, J.B., Gao, S., Sun, D., Li, H., Shu, X.X., and Yang, Z.M. (2013). miR394 and LCR are involved in Arabidopsis salt and drought stress responses in an abscisic acid-dependent manner. BMC Plant Biol. 13, 210

Sunkar, R., and Zhu, J.K. (2004). Novel and stress-regulated microRNAs and other small RNAs from Arabidopsis. Plant Cell 16, 2001-2019.

Sunkar, R., Kapoor, A., and Zhu, J.K. (2006). Posttranscriptional induction of two $\mathrm{Cu} / \mathrm{Zn}$ superoxide dismutase genes in Arabidopsis is mediated by downregulation of miR398 and important for oxidative stress tolerance. Plant Cell 18, 2051-2065.

Sunkar, R., Chinnusamy, V., Zhu, J., and Zhu, J.K. (2007). Small RNAs as big players in plant abiotic stress responses and nutrient deprivation. Trends Plant Sci. 12, 301-309.

Urao, T., Yamaguchi-Shinozaki, K., Urao, S., and Shinozaki, K. (1993). An Arabidopsis myb homolog is induced by dehydration stress and its gene product binds to the conserved MYB recognition sequence. Plant Cell 5, 1529-1539.

Vidal, E.A., Araus, V., Lu, C., Parry, G., Green, P.J., Coruzzi, G.M., and Gutiérrez, R.A. (2010). Nitrate-responsive miR393/AFB3 regulatory module controls root system architecture in Arabidopsis thaliana. Proc. Natl. Acad. Sci. USA 107, 4477-4482.

Voinnet, O. (2008). Post-transcriptional RNA silencing in plantmicrobe interactions: a touch of robustness and versatility. Curr. Opin. Plant Biol. 11, 464-470.

Voinnet, O. (2009). Origin, biogenesis, and activity of plant microRNAs. Cell 136, 669-687.

Yamaguchi-Shinozaki, K., and Shinozaki, K. (2006). Transcriptional regulatory networks in cellular responses and tolerance to dehydration and cold stresses. Annu. Rev. Plant Biol. 57, 781-803.

Yoo, J.H., Park, C.Y., Kim, J.C., Heo, W.D., Cheong, M.S., Park H.C., Kim, M.C., Moon, B.C., Choi, M.S., Kang, Y.H., et al. (2005). Direct interaction of a divergent CaM isoform and the transcription factor, MYB2, enhances salt tolerance in arabidopsis. J. Biol. Chem. 280, 3697-3706.

Yoshida, T., Fujita, Y., Sayama, H., Kidokoro, S., Maruyama, K., Mizoi, J., Shinozaki, K., and Yamaguchi-Shinozaki, K. (2010). AREB1, AREB2, and ABF3 are master transcription factors that cooperatively regulate $A B R E$-dependent $A B A$ signaling involved in drought stress tolerance and require $A B A$ for full activation. Plant J. 61, 672-685.

Zhao, B., Ge, L., Liang, R., Li, W., Ruan, K., Lin, H., and Jin, Y. (2009). Members of miR-169 family are induced by high salinity and transiently inhibit the NF-YA transcription factor. BMC Mol. Biol. 8, 10-29.

Zhao, M., Ding, H., Zhu, J.K., Zhang, F., and Li, W.X. (2011). Involvement of miR169 in the nitrogen-starvation responses in Arabidopsis. New Phytol. 190, 906-915.

Zhou, X., Wang, G., and Zhang, W. (2007). UV-B responsive microRNA genes in Arabidopsis thaliana. Mol. Syst. Biol. 3, 103

Zhou, X., Wang, G., Sutoh, K., Zhu, J.K., and Zhang, W. (2008). Identification of cold-inducible microRNAs in plants by transcriptome analysis. Biochim. Biophys. Acta. 1779, 780-788. 